\title{
Photon statistics in resonance fluorescence: results from an atomic-beam deflection experiment
}

\section{Citation for published version (APA):}

Hoogerland, M. D., Wijnands, M. N. J. H., Senhorst, H. A. J., Beijerinck, H. C. W., \& Leeuwen, van, K. A. H. (1990). Photon statistics in resonance fluorescence: results from an atomic-beam deflection experiment. Physical Review Letters, 65(13), 1559-1562. https://doi.org/10.1103/PhysRevLett.65.1559

DOI:

10.1103/PhysRevLett.65.1559

Document status and date:

Published: 01/01/1990

\section{Document Version:}

Publisher's PDF, also known as Version of Record (includes final page, issue and volume numbers)

\section{Please check the document version of this publication:}

- A submitted manuscript is the version of the article upon submission and before peer-review. There can be important differences between the submitted version and the official published version of record. People interested in the research are advised to contact the author for the final version of the publication, or visit the $\mathrm{DOI}$ to the publisher's website.

- The final author version and the galley proof are versions of the publication after peer review.

- The final published version features the final layout of the paper including the volume, issue and page numbers.

Link to publication

\section{General rights}

Copyright and moral rights for the publications made accessible in the public portal are retained by the authors and/or other copyright owners and it is a condition of accessing publications that users recognise and abide by the legal requirements associated with these rights.

- Users may download and print one copy of any publication from the public portal for the purpose of private study or research.

- You may not further distribute the material or use it for any profit-making activity or commercial gain

- You may freely distribute the URL identifying the publication in the public portal.

If the publication is distributed under the terms of Article 25fa of the Dutch Copyright Act, indicated by the "Taverne" license above, please follow below link for the End User Agreement:

www.tue.nl/taverne

Take down policy

If you believe that this document breaches copyright please contact us at:

openaccess@tue.nl

providing details and we will investigate your claim. 


\title{
Photon Statistics in Resonance Fluorescence: Results from an Atomic-Beam Deflection Experiment
}

\author{
M. D. Hoogerland, M. N. J. H. Wijnands, ${ }^{(a)}$ H. A. J. Senhorst, H. C. W. Beijerinck, \\ and K. A. H. van Leeuwen \\ Physics Department, Eindhoven University of Technology, P.O. Box 513, 5600 MB Eindhoven, The Netherlands \\ (Received 16 April 1990)
}

\begin{abstract}
Photon-number statistics in resonance fluorescence is studied through the deflection of a beam of neon atoms in the metastable ${ }^{3} P_{2}$ state by radiation pressure. An analysis of the deflection profile leads to experimental values for the Mandel $Q$ parameter as a function of laser intensity and detuning for different laser polarizations. The results confirm the predicted predominantly sub-Poissonian statistics. Good agreement is demonstrated with the results of a Monte Carlo calculation based on a pure-state analysis of resonance fluorescence.
\end{abstract}

PACS numbers: $42.50 . \mathrm{Bs}, 32.50 .+\mathrm{d}, 32.80 .-\mathrm{t}, 42.50 . \mathrm{Vk}$

The statistical properties of the spontaneous radiation emitted by a driven two-level atom have been extensively studied theoretically. Mandel ${ }^{\prime}$ pointed out the subPoissonian character of the distribution of the number of photons emitted in a given time and connected it to the well-known antibunching property evident in the distribution of the time interval between successive photon emissions. He introduced the normalized second factorial moment

$$
Q=\left[\left\langle(\Delta N)^{2}\right\rangle-\langle N\rangle\right] /\langle N\rangle
$$

as a measure of the deviation from Poissonian statistics, with $\langle N\rangle$ the average number of emitted photons and $\left\langle(\Delta N)^{2}\right\rangle$ the variance. Mandel derived a formula giving $Q$ as a function of interaction time $T$ and driving-field intensity. In the limit of long interaction time ( $T$ much larger than the natural lifetime $\tau$ of the upper level) a simple relation

$$
Q=-3 s /(1+s)^{2}
$$

is obtained, where the saturation parameter $s$ is proportional to the driving-field intensity $\left(s=\Omega^{2} / 8 A^{2}\right.$, with $\Omega$ the Rabi frequency and $A$ the Einstein coefficient for the transition). The minimum value, $Q=-\frac{3}{4}$, is obtained at $s=1$. This corresponds to a width of the photonnumber distribution equal to half the width of a Poissonian distribution.

Since then the theory has been extended to include detuning of the driving-field ${ }^{2}$ frequency and finite-bandwidth effects; 3,4 one of the results of the prediction of super-Poissonian statistics for large enough detuning. Analytical expressions for the complete photon-number distribution have been derived by Cook, ${ }^{5}$ Lenstra, ${ }^{6}$ and Kaminishi. $^{7}$

Experimentally, less information is available. The first experimental result has been reported by Short and Man$\mathrm{del},{ }^{8}$ who performed a photon-counting experiment using sodium as a two-level system with a dye laser to supply the driving field. The laser was on resonance and the light intensity was fixed at about half the saturation value $(s=0.5)$. They observed a negative value of $Q$ in good agreement with theory. Recently, more extensive results using essentially the same method have been reported for a transition between two levels in a three-level system prior to a quantum jump to the third level. ${ }^{9}$ The results confirmed Mandel's two-level formula [Eq. (1)]. A major problem in these photon-counting experiments is that the experimentally determined $Q$ parameter is equal to the theoretical value multiplied by the total detection efficiency. With a detection efficiency of, e.g., 0.0039 as in Mandel's experiment, deviations from Poissonian statistics are obviously strongly suppressed.

Cook $^{2}$ suggested studying photon statistics by analyzing the radiation-pressure-induced deflection of an atomic beam, using the simple relation between net momentum transfer and spontaneous emission. Essentially, the average deflection angle of the atoms in the beam is determined by the average number of spontaneously emitted photons and thus by the average upperstate population, while the spread in deflection angles is determined by the width of the photon-number distribution. An extensive theoretical analysis of light-induced deflection of an atomic beam, including both standingand running-wave cases, has been performed by Tanguy, Reynaud, and Cohen-Tannoudji. ${ }^{10}$ Several groups have announced such an experiment, ${ }^{11,12}$ but the only published results so far are the preliminary data of Wang et al., ${ }^{13}$ consisting of one measurement of $Q$ at $s \simeq 1.3$, coinciding well with theory.

We have performed an experiment along the lines of Cook's suggestion and report extensive results confirming the dependence of $Q$ on both light intensity and detuning from the transition frequency. The experiments are performed in an atomic-beam apparatus originally used for high-resolution total-cross-section measurements. ${ }^{14} \mathrm{~A}$ supersonic beam of ${ }^{20} \mathrm{Ne}$ atoms, partly excited in a discharge to the metastable $\{3 s\}{ }^{3} P_{2}$ state, is deflected by a laser beam (nearly) resonant with the transition to the $\{3 p\}^{3} D_{3}$ state $(\tau=19.4 \mathrm{~ns})$ at $640 \mathrm{~nm}$. This constitutes a closed-level system with only magnetic degeneracy. The atomic beam is collimated by $100 \times 500-$ and $50 \times 500$ $\mu \mathrm{m}$ slits positioned 5 and $1400 \mathrm{~mm}$ downstream of the 
source. The interaction region is located at $2200 \mathrm{~mm}$ from the source. The beam profile after deflection is analyzed using a stepper-motor-driven $50 \times 1000-\mu \mathrm{m}$ slit $760 \mathrm{~mm}$ downstream of the interaction region and an Auger-type metastable-atom detector. The beam is chopped to enable time-of-flight velocity analysis of the deflected atoms. This greatly simplifies the analysis, avoiding convolutions over the interaction time and longitudinal atomic momentum.

The laser beam, intersecting the atomic beam at right angles, is produced by a ring dye laser operating with DCM dye. ${ }^{15}$ It has a Gaussian waist (half width at $1 / e^{2}$ intensity) at the interaction region of $2.36 \mathrm{~mm}$; a 1.75 $\mathrm{mm}$ slit $0.3 \mathrm{~mm}$ before the interaction region produces an approximately flat-top intensity profile over the interaction region with $8 \% \mathrm{rms}$ variation. The interaction time ranges from 1.46 to $2.19 \mu$ s (corresponding to $75 \tau$ and $113 \tau$ ) for the $800-1200-\mathrm{ms}^{-1}$ experimental range of velocities, so that the long-time limit condition is fulfilled. The bandwidth of the laser is $\simeq 250 \mathrm{kHz}$, compared to the natural linewidth of $8 \mathrm{MHz}$, and has been neglected in the analysis.

An experimental deflected-beam profile for $1000-\mathrm{m} / \mathrm{s}$ atoms is shown in Fig. 1. The peak on the left results from the undeflected ${ }^{22} \mathrm{Ne}$ and ${ }^{21} \mathrm{Ne}$ atoms as well as ${ }^{20} \mathrm{Ne}$ atoms in the ${ }^{3} P_{0}$ metastable state. It serves as an excellent simultaneous calibration of the instrumental beam width and of the zero of the deflection scale. The solid line in Fig. 1 corresponds to the best fit with two Gaussian components; the width of the deflected beam is determined from the fitted profile in the rest of the analysis. The dashed line corresponds to a calculated curve assuming a Poissonian photon-number distribution. The difference in width immediately indicates the actual

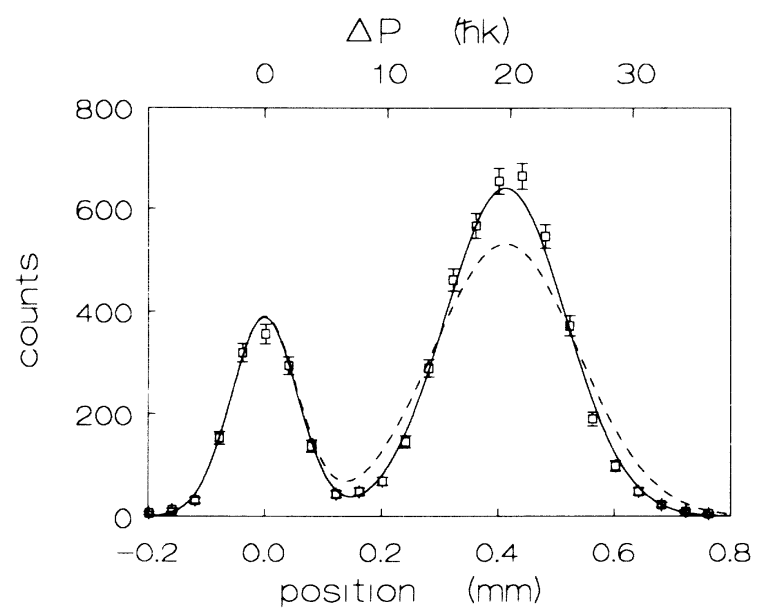

FIG. 1. Scan of the deflected-beam profile for $1000-\mathrm{m} / \mathrm{s}$ neon atoms. Squares: experimental data. Solid line: fit with two Gaussian peaks, leading to $Q=-0.58$ in the analysis. Dashed line: calculated curve assuming Poissonian statistics $(Q=0)$. On top, a momentum scale in units of a single-photon recoil momentum is shown.
sub-Poissonian character of the distribution.

Extracting the $Q$ parameter from the atomic momentum spread in the direction of the laser beam is straightforward using Eq. (2) from Ref. 2:

$$
\left\langle(\Delta P)^{2}\right\rangle=\left\langle\left(\Delta P_{0}\right)^{2}\right\rangle+(\hbar k)^{2}\left[\left\langle(\Delta N)^{2}\right\rangle+\alpha\langle N\rangle\right],
$$

which splits the momentum variance into contributions from the variance before deflection, from the variance of the photon-number distribution, and from the angular distribution of the spontaneous-emission-induced recoils. The factor $\alpha$ in the last contribution equals $\frac{2}{5}$ for a pure two-level system. In our case, $\alpha$ depends on the actual substate distribution. For the equilibrium distributions for circular and linear polarization, $\alpha$ equals $\frac{2}{5}$ and $\frac{16}{45}$, respectively. In view of the long interaction time in our experiments, the equilibrium values have been used.

The comparison of experimental results with theory is complicated by the lack of a pure two-state system due to magnetic degeneracy. Prepumping the atoms, using circularly polarized light, to the $M=2$ lower state would be best but cannot easily be done in our setup. Thus, the atoms enter the interaction region in a statistical mixture of all magnetic substates of the metastable level. During the interaction time, the atomic ensemble evolves towards a steady-state distribution which depends on the laser polarization; either a pure $M=2(M=-2)$ state with respect to the propagation direction of the laser beam for $\sigma^{+}\left(\sigma^{-}\right)$polarized light, or a 1:10:20:10:1 mixture of $M=-2, \ldots, 2$ with respect to the polarization direction for $\pi$ polarized light. This ensemble evolution is easily calculated by density-matrix techniques but the influence of the total photon number-and atomic momentum distributions-is much more difficult to evaluate.

The approach taken by us follows the Monte Carlo treatment of spontaneous emission and atomic momentum change used by Blatt et al. ${ }^{16}$ to model laser-cooling experiments. This approach is in turn based on Mollow's pure-state analysis of resonance radiation. ${ }^{17}$ Although Ref. 16 essentially treats a two-level system, extension in our case to include magnetic degeneracy is straightforward. The laser light, using either linear or circular polarization, couples each lower-level substate to only one upper-level substate and vice versa. Hence, starting from a randomly chosen lower-level magnetic substate, the coherent wave-function evolution will be that of a two-level system and the time to the next spontaneous emission can be randomly generated, as in Ref. 16.

Spontaneous emission induces a coupling to different lower-level substates. This coupling can be simply included by randomly choosing the lower-level substate after the emission of a photon, with the probability for each decay channel given by the appropriate branching ratios.

In Figs. 2(a) and 2(b) the on-resonance experimental results for the $Q$ parameter are shown together with the 


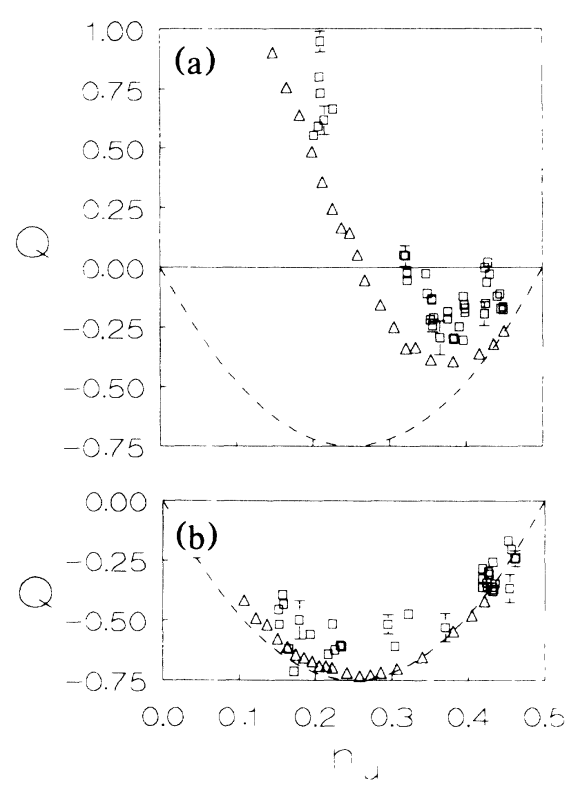

FIG. 2. Mandel $Q$ parameter as a function of average upper-state population $n_{u}$ for (a) $\sigma^{+}$and (b) $\pi$ polarization. Squares: values from experiment. Only a few representative error bars are shown. Triangles: results from Monte Carlo calculations. Dashed line: two-level formula [Eq. (4)].

results of the Monte Carlo calculation. Figure 2(a) shows the data for $\sigma^{+}$polarization and Fig. 2(b) shows those for $\pi$ polarization. Experimental results as well as Monte Carlo results have been obtained velocity resolved for the complete $800-1200-\mathrm{m} / \mathrm{s}$ velocity range and have been averaged afterward. The $Q$ parameter is shown as a function of the average upper-state population $n_{u}$ $=\langle N\rangle \tau / T$, as determined from the average beam deflection. For a two-level system, the intensity dependence of the upper-state population is given by $n_{u}=s / 2(s+1)$ and Mandel's formula leads to the simple quadratic expression

$$
Q=12 n_{u}\left(n_{u}-\frac{1}{2}\right) \text {. }
$$

The dependence of $n_{u}$ on the measured laser intensity has been separately checked and has been found to agree excellently with theory, allowing for an error in the absolute calibration of the laser power which is so far not understood.

Experimental results for both polarizations are seen to be in good agreement with the calculations, although the experimental results tend to be systematically somewhat too high. The agreement extends over the complete range of laser intensities (from $\simeq 20$ to $\simeq 800 \mu \mathrm{W}$ / $\mathrm{mm}^{2}$ ). A dramatic difference between the two polarizations is obvious. While for linear polarization the results are very close to the simple two-level, infiniteinteraction-time Mandel formula (see the dashed lines in Fig. 2), for circular polarization $Q$ is much higher. This is a direct consequence of the evolution from an equally populated statistical substate distribution towards a

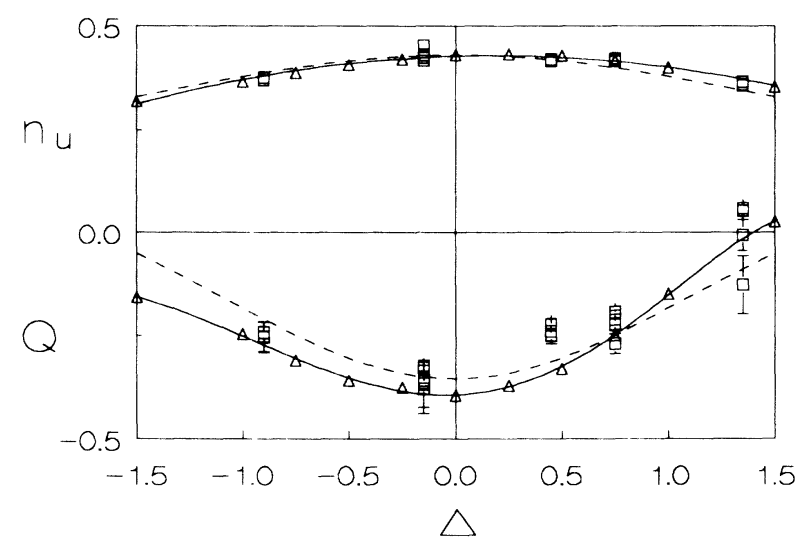

FIG. 3. $n_{u}$ (upper data) and $Q$ (lower data) as a function of normalized laser detuning $\Delta$. Squares: experimental values. Solid lines and triangles: results from Monte Carlo calculations. The dashed lines correspond to the two-level analytical formulas for $n_{u}$ and $Q$ [Eq. (5)].

steady-state distribution during the interaction time. For linear polarization, the time scale of the evolution is much smaller than the interaction time. The continuous hopping between our lower-level substates after equilibrium has been reached only introduces an effective averaging of the saturation parameter due to the different angular momentum factors in the transition moments. For circular polarization, due to the small transition rates out of the negative- $M$, states under $\sigma^{+}$excitation, the time scale of the evolution is of the same order as the interaction time except at very high laser intensities. This produces an extra inhomogeneous broadening effect which drastically increases the width of the photon-number and momentum distributions of the atomic ensemble. Another complication is the non-Gaussian character of this broadening effect, which is evident as an enhancement of the small-deflection tail of the Monte Carlo results. In the experimental results, this tail is masked by the undeflected peak. As a result, a correction has to be applied to the deflected-beam momentum variance as determined from the Gaussian-fit procedure. This correction (with a maximum of 0.5 in $Q$ at $n_{u}=0.2$ ) is taken.into account in the experimental results shown in Fig. 2(a).

The dependence of $n_{u}$ and $Q$ on the laser detuning $\delta \omega$ for linearly polarized light is shown in Fig. 3 . The results have been taken at one value of the laser intensity $\left(\simeq 400 \mu \mathrm{W} / \mathrm{mm}^{2}\right)$. The $n_{u}$ curve has been used to calibrate the detuning scale, which was not known absolutely. Again, calculation and experiment are in excellent agreement, demonstrating the rapid increase in $Q$ with increasing detuning. The asymmetry which is apparent in the curves is a consequence of the Doppler shift changing the effective laser detuning while the atomic beam is being deflected. While constituting only a small effect at zero detuning, the effect is more pronounced at larger initial detunings where the excitation rate varies 
appreciably with detuning. With "blue" detuning, the Doppler shift connected with the deflection moves the atoms closer to resonance, which increases $n_{u}$ and introduces a positive feedback in the deflection which increases the momentum spread and hence $Q$. The reverse is true for "red" detuning. In Fig. 3, Cook's analytical formula for $Q$ as a function of detuning,

$$
Q=\frac{-s\left(3-\Delta^{2}\right)}{\left(s+1+\Delta^{2}\right)^{2}}
$$

for a two-level system is shown as well (with $\Delta=2 \tau \delta \omega$ the normalized laser detuning). This curve does not show asymmetry.

Summarizing, accurate measurements on photonnumber statistics using the atomic-beam deflection technique suggested by Cook $^{2}$ have been performed. The predominantly sub-Poissonian character of the statistics has been confirmed. The $Q$ parameter has been measured for a wide range of driving-field intensity and for a number of values of the laser detuning. The experimental dependency on these parameters is in good agreement with the results of calculations based on a Monte Carlo approach of spontaneous emission, which enables us to include in a simple way the effects of magnetic degeneracy in the neon ${ }^{3} P_{2} \rightarrow{ }^{3} D_{3}$ closed-level system as well as those of finite interaction time and Doppler shift. For linear polarization, the results from the Monte Carlo calculation differ only slightly from the simple analytical formulas given by Cook and Mandel; for circular polarization, the differences are large, but agreement with ex- periment is still very good.

(a) Present address: C.V.I. N.V., P.O. Box 2233, 3500 GE Utrecht, The Netherlands.

${ }^{1}$ L. Mandel, Opt. Lett. 4, 205 (1979).

${ }^{2}$ R. J. Cook, Opt. Commun. 35, 347 (1980).

${ }^{3}$ S. Singh, Opt. Commun. 44, 254 (1983).

${ }^{4}$ H. F. Arnoldus and G. Nienhuis, Optica Acta 30, 1573 (1983)

${ }^{5}$ R. J. Cook, Phys. Rev. A 23, 1243 (1981).

${ }^{6}$ D. Lenstra, Phys. Rev. A 26, 3369 (1982).

${ }^{7}$ K. Kaminishi, J. Phys. Soc. Jpn. 53, 1006 (1983).

${ }^{8}$ R. Short and L. Mandel, Phys. Rev. Lett. 51, 384 (1983); R. Short and L. Mandel, in Coherence and Quantum Optics V, Proceedings of the Fifth Rochester Conference (Plenum, New York, 1984), p. 671.

${ }^{9}$ M. A. Finn, G. W. Greenlees, T. W. Hodapp, and D. A. Lewis, Phys. Rev. A 40, 1704 (1989).

${ }^{10}$ C. Tanguy, S. Reynaud, and C. Cohen-Tannoudji, J. Phys. B 17, 4623 (1984).

${ }^{11}$ K. Rubin and M. S. Lubell, Prog. Quant. Electron. 8, 219 (1984).

${ }^{12}$ B. Jaduszliwer, G. F. Shen, and B. Bederson, Phys. Rev. A 33, 3792 (1986).

${ }^{13}$ Y. Z. Wang, W. G. Huang, Y. D. Cheng, and L. Liu, in Laser Spectroscopy VII (Springer-Verlag, Berlin, 1985).

${ }^{14}$ E. R. T. Kerstel, C. P. J. W. van Kruysdijk, J. C. Vlugter, and H. C. W. Beijerinck, Chem. Phys. 121, 211 (1988).

154-(dicyanomethylene)-2-methyl-6( $p$-dimethyl-amino-styryl)$4 \mathrm{H}$-pryan is denoted DCM.

${ }^{16}$ R. Blatt, W. Ertmer, P. Zoller, and J. L. Hall, Phys. Rev. A 34, 3022 (1986).

${ }^{17}$ B. R. Mollow, Phys. Rev. A 12, 1919 (1975). 